\title{
BALANÇO DE ENERGIA E EVAPOTRANSPIRAÇÃO DO CAPIM MOMBAÇA SOB SISTEMA DE PASTEJO ROTACIONADO
}

\section{RODRIGO ALMEIDA MUNIZ ${ }^{1}$, ELIAS FERNANDES DE SOUSA ${ }^{2}$, JOSÉ CARLOS MENDONÇA², BARBARA DOS SANTOS ESTEVES ${ }^{2}$, LIDIANE DE LIMA LOUSADA ${ }^{2}$}

\author{
${ }^{1}$ Universidade São Paulo, Escola Superior de Agricultura Luiz de Queiroz (USP/ESALQ), Piracicaba, Brasil \\ ${ }^{2}$ Universidade Estadual do Norte Fluminense Darci Ribeiro, Centro de Ciências e Tecnologias \\ Agropecuárias, Laboratório de Engenharia Agrícola (CCTA/LEAG), Campos dos Goytacazes, RJ, Brasil
}

muniz.ra@usp.br, efs@uenf.br, mendonca@uenf.br, barbbarase@yahoo.com.br, delimalousada@yahoo.com.br

Recebido Setembro de 2011 - Aceito Maio de 2013

\begin{abstract}
RESUMO
No cenário atual das mudanças climáticas, estudos apontam que a pecuária é uma das maiores responsáveis pelas emissões de Gases de Efeito Estufa (GEE) devido ao desmatamento para a expansão das áreas de pasto. Frente a esse quadro, a irrigação de pastagens pode intensificar a utilização das pastagens. No entanto, para um manejo adequado da irrigação é de fundamental importância a determinação da evapotranspiração para subsidiar as decisões sobre quando, como e quanto irrigar, evitando consumo de água e energia desnecessários e outros problemas relacionados ao manejo inadequado da irrigação. Neste contexto, objetivou-se quantificar o balanço de energia pelo método da razão de Bowen e evapotranspiração do capim Mombaça irrigado sob sistema de pastejo rotacionado localizado na unidade experimental da Universidade Estadual do Norte Fluminense Darcy Ribeiro no Município de Campos dos Goytacazes, RJ. Instalou-se uma estação micrometeorológica automática em uma área de pastagem, que mediu os fluxos de energia sobre a cultura no período de outono/ inverno de 2010. Os fluxos de calor latente, sensível e no solo representaram em média durante o período 66,23 e $12 \%$ do saldo de radiação, respectivamente. A evapotranspiração média do capim Mombaça irrigado foi 3,87 $\mathrm{mm} \mathrm{dia}^{-1}$, a máxima de $8,93 \mathrm{~mm} \mathrm{dia}^{-1} \mathrm{e}$ a mínima de $0,43 \mathrm{~mm} \mathrm{dia}{ }^{-1}$.

Palavras-chave: micrometeorologia, recursos hídricos, Panicum maximum.
\end{abstract}

\begin{abstract}
ENERGY BALANCEAND EVAPOTRANSPIRATION OF THE GRASS MOMBAÇA under ROTATED PASTURE SYSTEM

In the current scenario of climate changes, researches indicate that the livestock is one the of highest activities contributing to the Greenhouse Gases Emissions (GGE) due to deforestation to expand the pasture areas. In front of this scenario, the irrigation of pasture can to intensify the use of the pasture. However the appropriate management of the irrigation requires the determination of the evapotranspiration to subsidize the decisions about when, how and how much to irrigate avoiding excess consumption of water and energy and other problems related to inadequate irrigation management. In this context, it is aimed to quantify the energy balance by Bowen Ratio method and the evapotranspiration of Mombaça grass irrigated under rotated pasture system at the experimental site of the Universidade Estadual do Norte Fluminense Darcy Ribeiro, in the city of Campos dos Goytacazes, RJ. An automatic micrometeorological station on the pasture area was installed, measuring the fluxes of energy over the crop during the 2010 fall/winter period. On average, the fluxes of latent heat, sensible heat and soil heat represented 66,23 and $12 \%$ of the net radiation, respectively. The average evapotranspiration of the grass Mombaça was $3.87 \mathrm{~mm}^{-1 a y}{ }^{-1}$, with a maximum of $8.93 \mathrm{~mm}$ day $^{-1}$ and a minimum of $0.43 \mathrm{~mm}$ day $^{-1}$.
\end{abstract}

Keywords: micrometeorology, water resources, Panicum maximum. 


\section{INTRODUÇÃO}

Atualmente sabe-se que o desflorestamento e as mudanças de uso do solo são grandes fontes de emissão de gases de efeito estufa (Broich et al., 2011; Cerbu et al., 2011; Gallardo e Bond, 2011). Desta forma, a atividade pecuária encontra-se sob questionamento quanto a sua sustentabilidade ambiental, sendo considerada uma das principais responsáveis pelo desmatamento.

Segundo Dias Filho e Andrade (2005) a Amazônia Legal possui uma área de 5,2 milhões de $\mathrm{km}^{2}$ e em 2007 havia uma área acumulada de desmatamento de $691.123 \mathrm{~km}^{2}$. De toda a área desmatada da Amazônia Legal, 80\% são utilizadas como pastagem e destas, 50\% encontravam-se degradadas. Além da emissão causada pelo desflorestamento, Pedreira et al. (2005) e Nusbaum (2010) citam que os ruminantes convertem os carboidratos presentes na sua dieta em ácidos graxos voláteis, resultando na produção de dióxido de carbono e metano. Por isso, os ruminantes são reconhecidos como importantes emissores de gases de efeito estufa.

Uma das consequências do aquecimento global é o possível impacto negativo sobre a produtividade das culturas (Zhou et al., 2010; Lal, 2011; Mengu et al., 2011). Diante deste cenário, o aumento da produção agrícola mundial é inevitável, necessitando de expansão das fronteiras agrícolas, a fim de suprir a demanda mundial de alimentos. Sparovek et al. (2011) citam que no Brasil, a agropecuária ocupa 275 Mha, sendo a maior parte utilizada com pastagens (211 Mha).

Aliado ao aumento da produção agrícola, Rivero et al. (2009) citam que a demanda interna por toneladas equivalentes de carcaça será de 7,5 milhões até 2012, aumentando a pressão da pecuária sobre as áreas de floresta, devido à baixa densidade dos rebanhos na região da Amazônia Legal que é em torno de uma cabeça por hectare. Os autores citam ainda a necessidade da intensificação da atividade, ou seja, deve-se lançar mão de técnicas que aumentem a produtividade das forragens e consequentemente o aumento da unidade animal por hectare. Experimento realizado por Ribeiro et al. (2009) mostrou que pastagens irrigadas têm grande efeito sobre a taxa de lotação do pasto, podendo chegar a 4,5 e 4,9 animais/ha na época seca em pastagem de capim Elefante e Mombaça, respectivamente.

No Brasil, a irrigação de pastagens não tem sido feita de maneira adequada, levando a aplicação excessiva de água, o que resulta em prejuízos ao ambiente, consumo desnecessário de energia elétrica e de água, lixiviação de nutrientes e maior compactação do solo, repercutindo na diminuição da produção e vida útil das pastagens. Para o manejo adequado da irrigação é preciso saber como, quanto e quando irrigar. Para tanto, a determinação da evapotranspiração é de fundamental importância em pastagens irrigadas, pois estabelece o consumo de água e, por consequência, a lâmina de água a ser aplicada pelo sistema (Alencar et al., 2009).

Há vários métodos para a determinação da evapotranspiração, porém a escolha por um método específico depende de uma série de fatores, tais como: da disponibilidade de dados meteorológicos e da escala de tempo desejada. Métodos complexos, como o de Penman - Monteith, que apesar de ser adotado pela Food and Agriculture Organization (FAO) como o método padrão de estimativa da ETo na escala diária, exigem grande número de variáveis meteorológicas e, por isso, têm aplicação limitada, somente sendo utilizados quando há disponibilidade de todos os dados necessários (Mendonca e Dantas, 2010).

O método da razão de Bowen para a determinação da evapotranspiração real, pode ser obtido independente das condições do tempo e não requer informações sobre as características aerodinâmicas da superfície experimental. No entanto, qualquer imprecisão instrumental refletirá na medida dos fluxos de calor latente, de calor sensível e de calor no solo. Outra desvantagem do método é que com valores da razão de Bowen próximos a menos um os fluxos de calor tornam-se inconsistentes, situação que normalmente ocorre ao amanhecer e ao entardecer, quando os fluxos de calor sensível e de calor latente são de magnitude aproximada, mas em direções opostas (Shi et al., 2008)2008.

$\mathrm{O}$ método do balanço de energia, baseado na razão de Bowen, constitui-se em um modelo simples e prático de estimativa do fluxo de calor latente, que vem sendo amplamente utilizado para a determinação da evapotranspiração (Ünlü et al., 2010; Guo e Sun, 2011; Lima et al., 2011; Silva et al., 2011). Neste contexto, objetivou-se com a pesquisa quantificar os componentes do balanço de energia à superfície e a evapotranspiração do capim Mombaça utilizando o método da razão de Bowen, sob uma área de pastejo rotacionado localizada em Campos dos Goytacazes, RJ.

\section{MATERIAL E MÉTODOS}

\section{1 Área de estudo}

O estudo foi realizado na Escola Técnica Estadual Agrícola Antônio Sarlo, no Município de Campos dos Goytacazes, região norte do estado do Rio de Janeiro, estando a sede do município localizada nas coordenadas $21^{\circ} 45^{\prime} 14^{\prime \prime} \mathrm{S}$ e $41^{\circ} 19^{\prime} 26^{\prime}$ 'W. Segundo a classificação de Köppen, o clima é do tipo Aw, ou seja, tropical úmido com verão chuvoso e inverno seco, sendo a temperatura do mês mais frio superior a $18{ }^{\circ} \mathrm{C}$. As médias anuais de precipitação, de temperatura e de umidade relativa do ar são respectivamente iguais a $1023 \mathrm{~mm}$, $24^{\circ} \mathrm{C}$ e $77 \%$ 
Aárea de estudo é ocupada por uma pastagem homogênea de capim Mombaça (Panicum maximum cv. Mombaça) com um ano de plantio, localizada na unidade experimental da Universidade Estadual do Norte Fluminense Darcy Ribeiro (UENF), situada na Escola Técnica Estadual Agrícola Antônio Sarlo, com aproximadamente nove ha, dividida em piquetes com cerca de $2500 \mathrm{~m}^{2}$, cujas coordenadas são $21^{\circ} 42^{\prime} 35,22^{\prime \prime} \mathrm{S}$ e $41^{\circ} 20^{\prime} 11,75^{\prime \prime} \mathrm{W}$.

A pastagem é irrigada por um sistema de irrigação por aspersão em malha, cujo funcionamento efetivo se dá no período de estiagem, a fim de manter o solo próximo à capacidade de campo. O solo da área possui uma composição textural com $57,5 \%$ de argila, $35 \%$ de silte e $7,5 \%$ de areia. A densidade do solo e de partícula é respectivamente de $1,15 \mathrm{~g} \mathrm{~cm}^{-3}$ e 2,51 $\mathrm{g} \mathrm{cm}^{-3}$ na camada de $0-20 \mathrm{~cm}$. O solo possui a capacidade de campo de $50 \%$ e ponto de murcha permanente de $25 \%$, sendo a disponibilidade de água igual a $25 \%$ de umidade volumétrica.

A intensidade de pastejo ou carga animal por piquete é variável, sendo função da disponibilidade de forragem, mantendo-se a pressão de pastejo de $4 \mathrm{~kg}$ de matéria seca foliar por $100 \mathrm{~kg}$ de peso vivo animal.

Para determinação da taxa de lotação no pré-pastejo avaliou-se a disponibilidade de forragem do piquete. O tempo máximo de pastejo foi de quatro dias, com um período de descanso de 28 dias, totalizando um ciclo de 32 dias entre os pastejos. Neste sistema de manejo o resíduo deixado após o pastejo varia entre 15 e $30 \mathrm{~cm}$, dependendo da estrutura do dossel. Após a retirada dos animais foram realizadas adubações de suplementação, com $50 \mathrm{~kg} \mathrm{ha}^{-1}$ de sulfato de amônia e 20 $\mathrm{kg} \mathrm{ha}^{-1}$ de cloreto de potássio. Foram avaliados três ciclos de pastejo nos meses de abril a setembro de 2010, que corresponde ao período do final das estações do outono e de inverno.

\subsection{Instrumentação utilizada}

Foi instalado em um piquete central da área uma estação micrometeorológica contendo os seguintes sensores: 1 saldo radiômetro (NR Lite); 2 piranômetros LI 200 (X-Li-cor); 1 anemômetro Met One (RM Young); 3 fluxímetros HFP01SC-L (Hukseflux) e 2 conjuntos psicrométricos, equipados com sensores de temperatura, que medem a temperatura de bulbo úmido e bulbo seco em sistema de circulação forçada, através da instalação de dois coolers mantidos a bateria. As hastes horizontais foram dispostas sobre o dossel da cultura a $0,10 \mathrm{~m}$ $\left(1^{\mathrm{a}}\right.$ haste) e a $1,0 \mathrm{~m}$ ( $2^{\mathrm{a}}$ haste), as quais foram elevadas de acordo com o crescimento da cultura segundo metodologia proposta por Silva et al. (2005). Os dados foram coletados a cada minuto e armazenados em valores médios a cada 15 minutos por um coletor de dados (datalogger) CR10X da Campbell Scientific Inc., Logan, Utah, USA. Foi instalado ainda um pluviômetro registrador da Squitter do Brasil, com 0,25 mm de sensibilidade.

\subsection{Precipitação e umidade do solo}

A precipitação pluviométrica do período do experimento está mostrada na Figura 1. Observa-se que os eventos de precipitação foram bem distribuídos até meados do $3^{\circ}$ ciclo de pastejo. A precipitação total foi de 26,6, 63,4 e 66,2 mm durante o $1^{\circ}, 2^{\circ}$ e $3^{\circ}$ ciclos de pastejo, respectivamente. No entanto, a cultura apresentou déficit hídrico apenas no final do $3^{\circ}$ ciclo de pastejo, devido a problemas ocorridos no sistema de irrigação o que inviabilizou as irrigações, conforme mostrado na Figura 2. O armazenamento médio de água no solo foi de $33,6 \mathrm{~mm}$, sendo o mínimo de $16,4 \mathrm{~mm}$ no dia Juliano 244 e o máximo de 51,8 mm no dia Juliano 202, que correspondem ao $3^{\circ}$ ciclo de pastejo.

\subsection{Estimativa do balanço de energia pelo método da razão de Bowen}

O saldo de radiação influencia diretamente na evapotranspiração das plantas. Segundo o princípio da conservação de energia, o saldo de radiação pode ser calculado de acordo com:

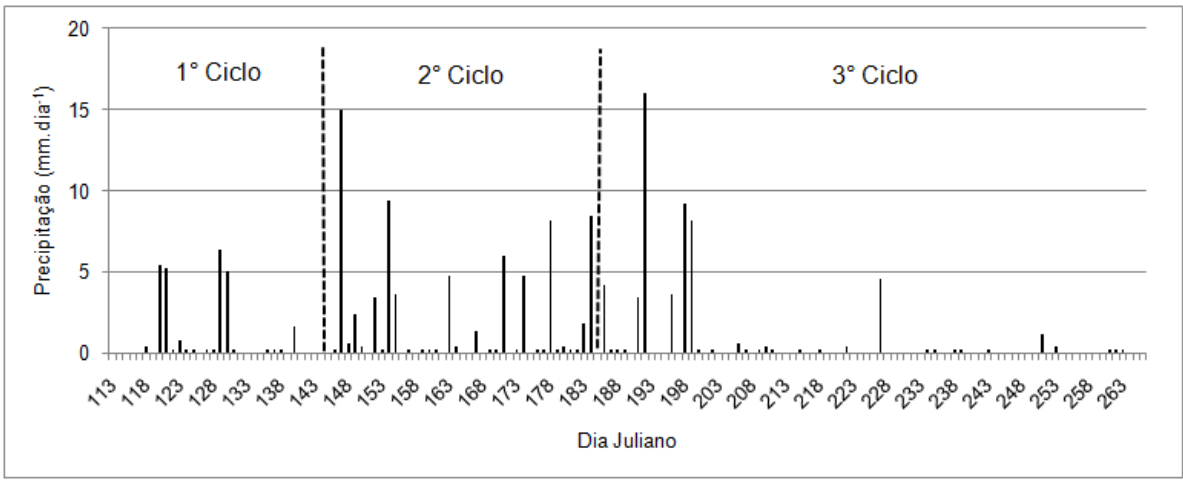

Figura 1. Eventos de precipitação pluvial diária sobre pastagem de capim Mombaça localizado na Escola Técnica Estadual Agrícola Antônio Sarlo no período de outono/inverno de 2010. 


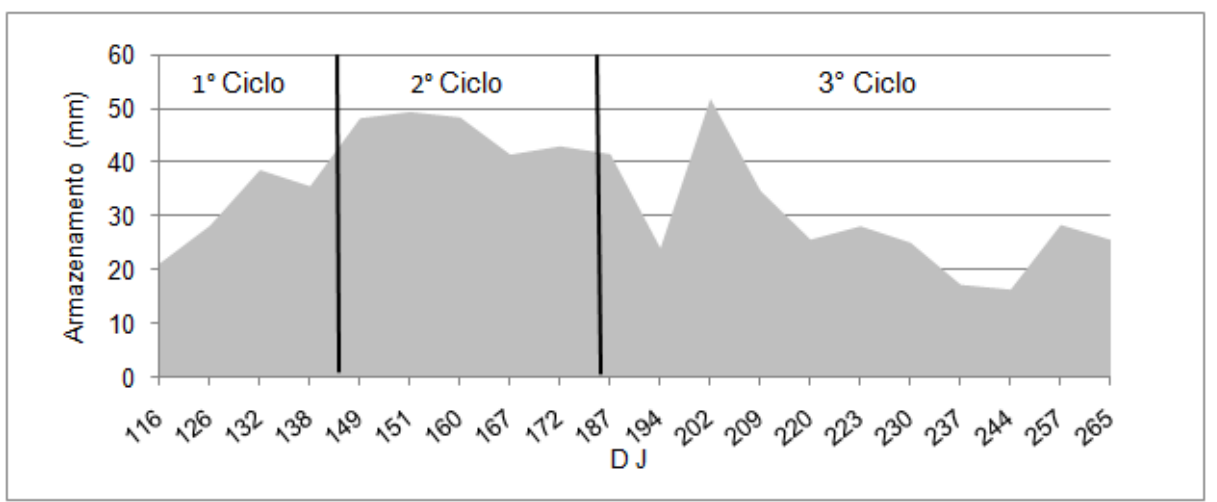

Figura 2 - Variação do armazenamento de água na camada de 0-20 cm do solo em função do Dia Juliano (DJ) durante três ciclos de pastejo do capim Mombaça.

$$
\mathrm{R}_{\mathrm{n}}-\mathrm{G}=\mathrm{H}+\lambda \mathrm{E}
$$

em que $\mathrm{R}_{\mathrm{n}}$ é o saldo de radiação, $\mathrm{G}$ é o fluxo de calor no solo, $\mathrm{H}$ é o fluxo de calor sensível e $\lambda \mathrm{E}$ é o fluxo de calor latente, todos em $\mathrm{MJ} \mathrm{m}^{-2} \mathrm{~h}^{-1}$.

A razão entre os fluxos de calor sensível e de calor latente foi proposta por Bowen (1926) como uma forma de análise da partição da energia disponível, sendo esta razão representada pela letra grega $\beta$ (beta) e obtida de acordo com a equação:

$$
\beta=\frac{\mathrm{H}}{\lambda \mathrm{E}}
$$

A Equação 2 pode ser reescrita da seguinte forma:

$$
\beta=\frac{\gamma * \Delta \mathrm{T}}{\Delta \mathrm{e}}
$$

em que $\gamma$ é o coeficiente psicrométrico, cujo valor assumido é de $0,0626 \mathrm{kPa} .{ }^{\circ} \mathrm{C}^{-1}, \Delta \mathrm{T}$ é a diferença de temperatura do ar em dois níveis em ${ }^{\circ} \mathrm{C}$ e $\Delta$ e é a diferença de pressão de vapor d'água em dois níveis dada em $\mathrm{kPa}$.

A pressão de vapor d'água do ar saturado $\left(\mathrm{e}_{\mathrm{s}}\right) \mathrm{em} \mathrm{kPa}$ foi calculado por (Murray, 1967):

$$
e_{s}=0,6108 \exp ^{\left[\frac{17,27 T_{a}}{237,3+T_{a}}\right]}
$$

A pressão de vapor d'água atual do ar $\left(\mathrm{e}_{\mathrm{a}}\right)$ em $\mathrm{kPa}$ foi calculada em função da umidade relativa em cada nível através de:

$$
\mathrm{e}_{\mathrm{a}}=\frac{\mathrm{UR} \% * \mathrm{e}_{\mathrm{s}}}{100}
$$

O fluxo de calor latente $(\lambda E)$, em $\mathrm{MJ} \mathrm{m}^{-2} \mathrm{~h}^{-1}$ foi calculado pela equação:

$$
\lambda \mathrm{E}=\frac{\mathrm{Rn}-\mathrm{G}}{(1+\beta)}
$$

em que $R_{n}$ é o saldo de radiação e $G$ o fluxo de calor no solo, ambos em $\mathrm{MJ} \mathrm{m}^{-2} \mathrm{~h}^{-1}$.
A evapotranspiração do capim Mombaça - ET (mm) foi calculada em função do fluxo de calor latente através da equação:

$$
\mathrm{ET}=\frac{\lambda \mathrm{E}}{\lambda}
$$

onde $\lambda\left(2,45 \mathrm{MJ} \mathrm{kg}^{-1}\right)$ é o calor latente de evaporação da água. O fluxo de calor sensível $(\mathrm{H})$ em $\mathrm{MJ} \mathrm{m}^{-2} \mathrm{~h}^{-1}$ foi obtido pelo produto da razão de Bowen $(\beta)$ e o fluxo de calor latente $(\lambda \mathrm{E})$ em $M J \mathrm{~m}^{-2} \mathrm{~h}^{-1}$. Para a realização dos cálculos da razão de Bowen trabalhou-se com médias horárias do período diurno, no intervalo entre 6-18 h. A fim de tornar os resultados da razão de Bowen mais consistentes, foi aplicado aos dados um filtro com o objetivo de excluir dados errôneos de acordo com a metodologia proposta por Silva et al. (2011).

Foram selecionados quatro dias representativos para a visualização e análise do saldo de radiação e dos fluxos de calor latente, calor sensível e calor no solo. Foram selecionados dias de céu claro sendo um dia para o $1^{\circ}$ e $2^{\circ}$ ciclo de pastejo e dois dias para o $3^{\circ}$ ciclo de pastejo.

\section{RESULTADOS E DISCUSSÃO}

\subsection{Balanço de energia}

No período de estudo, a temperatura média foi de $21^{\circ} \mathrm{C}$ e a umidade relativa média foi de $84,5 \%$. A temperatura e umidade relativa média do ar no $1^{\circ}, 2^{\circ}$ e $3^{\circ}$ ciclos de pastejo foram 22,2 , 20,7 e $21,25^{\circ} \mathrm{C}$ e 80,88 e $83 \%$, respectivamente. A irradiância solar global média no período foi de $28,57 \mathrm{MJ} \mathrm{m}^{-2}$, enquanto que o saldo de radiação médio foi de $13,62 \mathrm{MJ} \mathrm{m}^{-2}$, correspondendo a cerca de $41 \%$ da irradiância solar global do período.

A Figura 3 apresenta a variação diária dos fluxos de energia sobre a pastagem do capim Mombaça, durante os três ciclos de pastejo. Durante o período os componentes do balanço de energia foram em média $66 \%$ de $\lambda \mathrm{E}, 12 \%$ de $\mathrm{G}$ e $23 \%$ de $\mathrm{H}$, sendo o fluxo de calor latente a maior fração do saldo de radiação, 


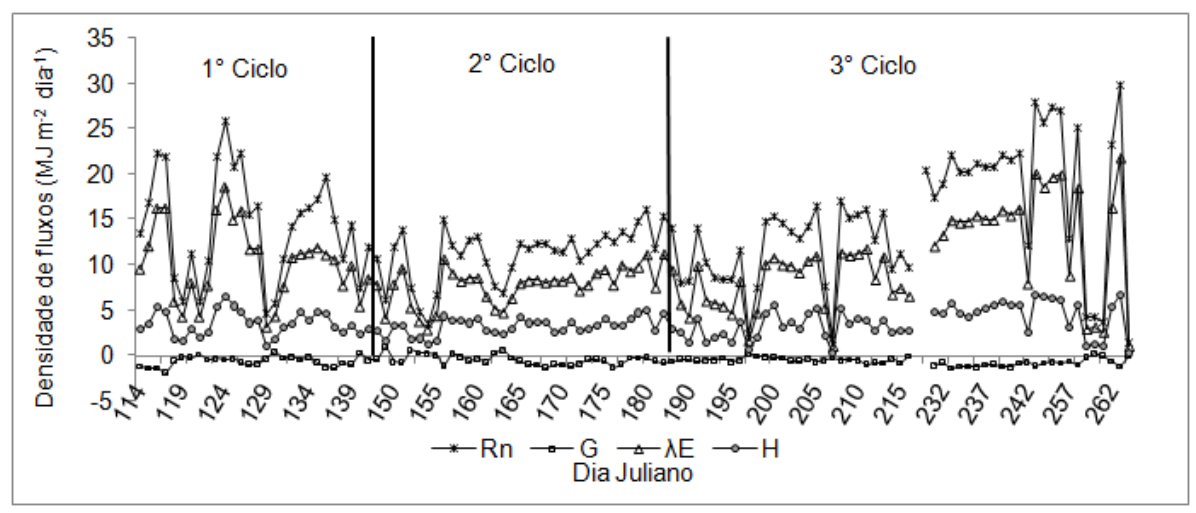

Figura 3 - Densidade dos fluxos de calor latente $(\lambda \mathrm{E})$, calor sensível $(\mathrm{H})$ e de calor no solo $(\mathrm{G})$ e o saldo de radiação (Rn) sobre pastagem do capim Mombaça em função do dia Juliano (DJ) durante três ciclos de pastejo rotacionado, em Campos dos Goytacazes, RJ.

enquanto que o fluxo de calor no solo apresentou a menor; porém, este obteve maior variação ao longo dos ciclos devido às sucessivas desfolhações provocadas pelo pastejo animal que reduz o IAF, aumentando a irradiância solar incidente sobre o solo, conforme pode ser observado pelos coeficientes de variação (CV) que foram de 6, 32 e 9\% para os fluxos de calor latente, calor no solo e calor sensível, respectivamente. Resultados semelhantes também foram encontrados por Mendonça et al. (2007) e André et al. (2010) que observaram redução do fluxo de calor no solo com aumento do IAF nas culturas do feijão e da cana-de-açúcar, ambos em Campos dos Goytacazes, RJ.

O fluxo de calor latente (média $=9,48 \mathrm{MJ} \mathrm{m}^{-2} \mathrm{dia}^{-1}$ ) foi maior que os fluxos de calor sensível (média $=3,36 \mathrm{MJ} \mathrm{m}^{-2}$ $\mathrm{dia}^{-1}$ ) e calor no solo (média $=-0,59 \mathrm{MJ} \mathrm{m}^{-2} \mathrm{dia}^{-1}$ ) durante todo o período de avaliação devido à disponibilidade de água no solo, proveniente da chuva e irrigação. Com água disponível no sistema maior quantidade de energia é alocada para a evapotranspiração (Lima et al., 2011; Silva et al., 2011).

A Figura 4 apresenta a variação da razão de Bowen ( $\beta)$ no período diurno dos dias característicos. Os valores médios de $\beta$ foram de $0,37,0,47,0,32$ e 0,33 para os dias Juliano 131, 167,187 e 237 , respectivamente. Esses valores mostram que a maior parte da energia foi disponibilizada para o processo evapotranspirativo do sistema e que o solo estava sob condições de umidade adequada para suprir a demanda hídrica da planta. Os valores encontrados estão na mesma ordem de grandeza de trabalho sobre a evapotranspiração de pastagens na região da Amazônia (Biudes et al., 2008).

Na Tabela 1 estão apresentadas as médias dos fluxos e a evapotranspiração média diária do capim Mombaça nos dias característicos. Os resultados apresentados refletem a variação dentro das estações do ano. Percebe-se uma redução do $R_{n}$ do dia 131 para do dia 167 devido a estação do ano em que foram medidos, outono e inverno respectivamente. Entre os dias 167 e 237 os valores de $R_{n}$ foram crescentes devido a aproximação da primavera, período em que naturalmente ocorre um aumento da irradiância solar global. A razão entre $\lambda E / R_{n}$ foi $0,76,0,68$ e 0,73 ; a razão entre $H / R_{n}$ foi de 0,24 , 0,40 e 0,30 e a razão entre $G / R_{n}$ foi de $0,01,0,08$ e 0,05 para os dias Juliano 131, 167, 187 e 237, respectivamente.

\subsection{Evapotranspiração da cultura}

A Figura 5 apresenta a variação da evapotranspiração do capim Mombaça durante os três ciclos de pastejo. Observa-se que a evapotranspiração da cultura sofreu variação sazonal dependente da radiação disponível no período. A estação do ano com menor saldo de radiação, o inverno, coincidiu com a menor evapotranspiração; com a proximidade da primavera (fim do inverno) e consequentemente maior incidência solar da estação, a evapotranspiração aumentou. A variabilidade diária, com picos ascendentes e descendentes, corresponde a dias nublados. A evapotranspiração média do período foi 3,93 $\mathrm{mm} \mathrm{dia}{ }^{-1}$ e o desvio padrão de $1,81 \mathrm{~mm}$. A evapotranspiração máxima foi de $8,93 \mathrm{~mm} \mathrm{dia}{ }^{-1}$ e a mínima de $0,43 \mathrm{~mm} \operatorname{dia}^{-1}$. Valores semelhantes foram encontrados por Silva et al. (2005)

Tabela 1 - Média diária dos fluxos de energia em MJ m-2 dia-1 e evapotranspiração média diária em $\mathrm{mm}$ dia-1 para os dias característicos, sobre a cultura do capim Mombaça em sistema de pastejo rotacionado no período de outono/inverno

Fluxo de Energia Dia 131 Dia 167 Dia 187 Dia 237

\begin{tabular}{ccccc}
\hline $\mathrm{R}_{\mathrm{n}}{ }^{*}$ & 14,18 & 12,29 & 15,28 & 20,71 \\
$\lambda \mathrm{E}^{*}$ & 10,88 & 8,38 & 11,25 & 15,03 \\
$\mathrm{H}^{*}$ & 3,47 & 4,95 & 4,59 & 5,75 \\
$\mathrm{G}^{*}$ & $-0,18$ & $-1,04$ & $-0,77$ & $-1,07$ \\
$\mathrm{ET}^{* *}$ & 3,11 & 3,42 & 4,59 & 6,13 \\
\hline
\end{tabular}

*unidade em MJ m-2 dia-1. ** unidade em mm dia-1 

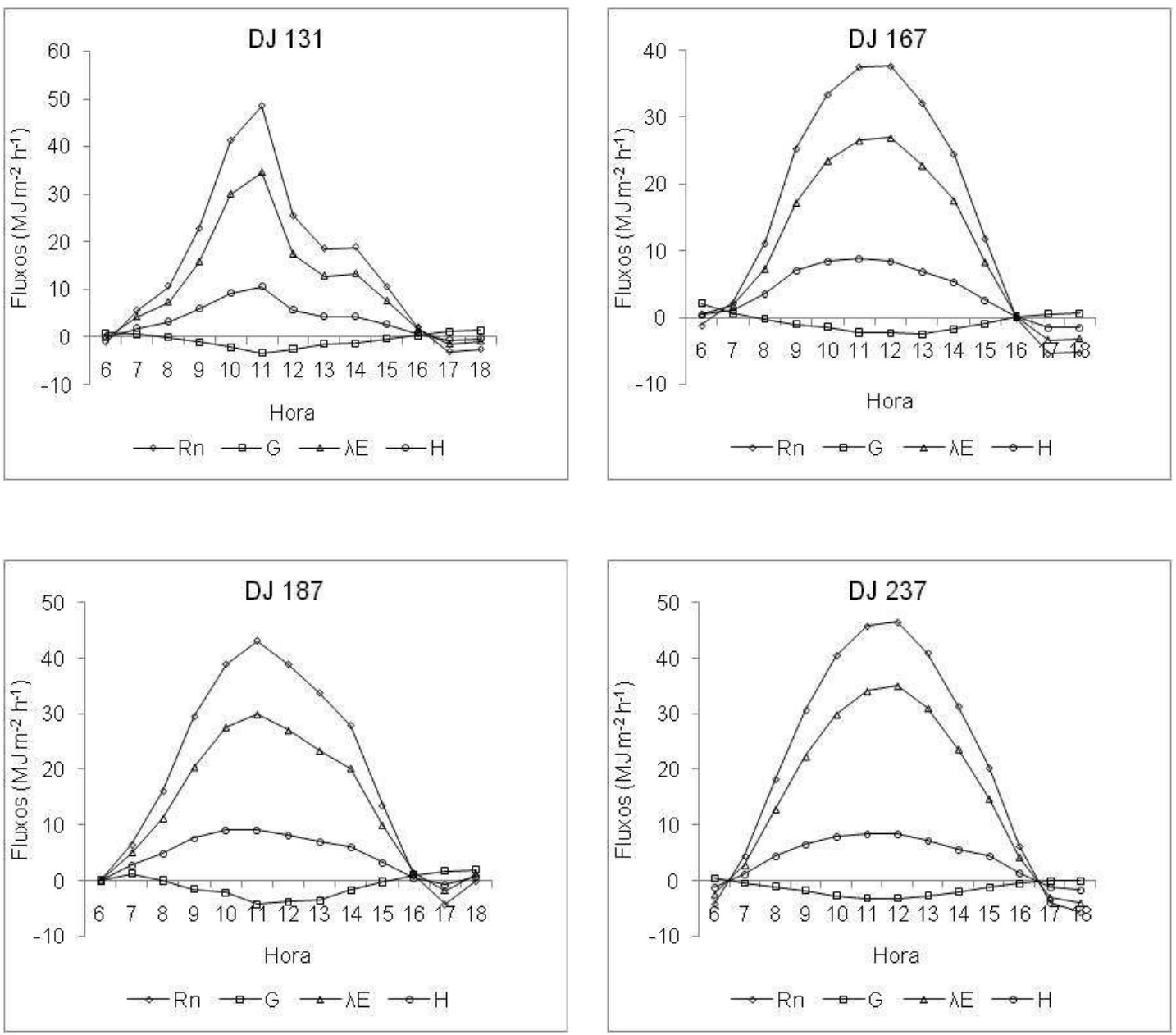

Figura 4 - Densidade dos fluxos do balanço de energia sobre pastagem de capim Mombaça para os dias característicos de 131, 167, 187 e 237, obtidos pela razão de Bowen.

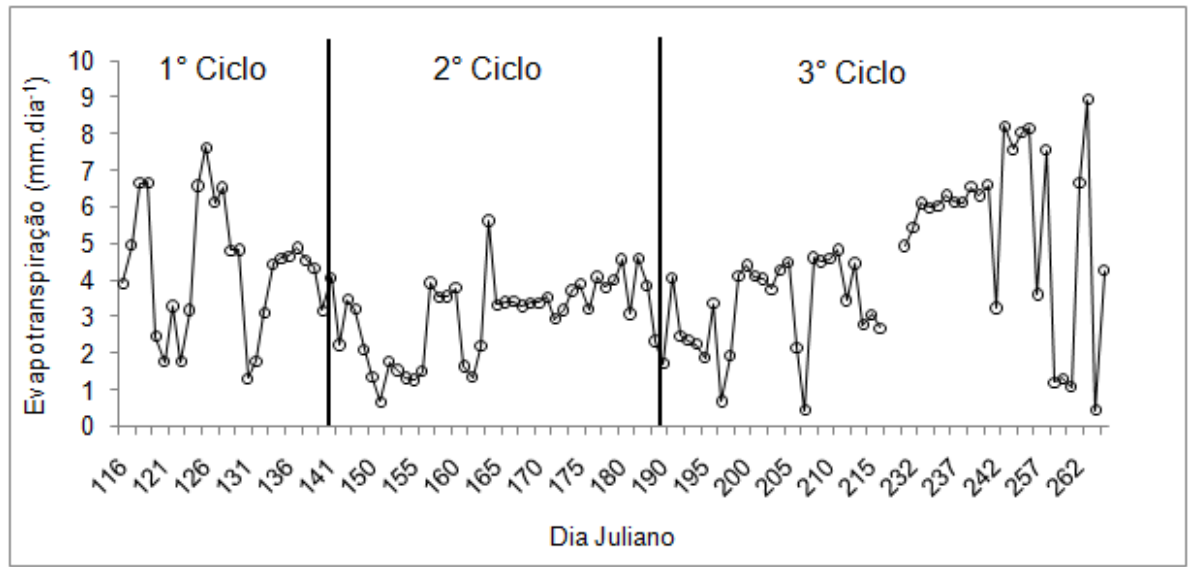

Figura 5 - Evapotranspiração do capim Mombaça em mm dia-1 sob sistema de pastejo rotacionado no período de outono/inverno em Campos dos Goytacazes, RJ. 
em Piracicaba, SP no período de junho e julho para o capim Tanzânia. Esses autores obtiveram uma evapotranspiração de $4,34 \mathrm{~mm} \mathrm{dia}^{-1}$, enquanto que Delgado-Rojas et al. (2004) sob mesmas condições observaram uma evapotranspiração nos meses de agosto a novembro entre 0,70 e $5,33 \mathrm{~mm} \mathrm{dia}^{-1}$ sendo os maiores valores registrados no mês de novembro.

A evapotranspiração da cultura não sofreu variação dentro de um ciclo de pastejo que pudesse ser indicativo de diferentes estádios de rebrota do capim Mombaça, mesmo após pastejo no qual há uma drástica redução do índice de área foliar. Isto ocorreu porque em um sistema de pastejo rotacionado, há piquetes com diferentes tempos de descanso, que resulta em diferentes índices de área foliar. Segundo Heilman et al. (1989) o balanço de energia para a estimativa dos fluxos de calor pelo método da razão de Bowen requer uma distância mínima de fetch que pode variar de 1:20 a 1:100 $\mathrm{m}$. Como o piquete possui aproximadamente 20 $\mathrm{m}$ de largura, a estação micrometeorológica estava a $10 \mathrm{~m}$ de distância de piquetes ao lado com índices de área foliar maior. Houve, no entanto, contribuição de umidade proveniente da evapotranspiração de piquetes vizinhos, por não haver uma área suficiente para atender a distância mínima de fetch exigida. Portanto, para a determinação da evapotranspiração na altura do piquete seria necessário lançar mão de outros métodos para a estimativa da evapotranspiração.

\section{CONCLUSÕES}

Os fluxos de calor latente, calor sensível e calor no solo representaram em média durante o período 66,23 e $12 \%$ do saldo de radiação.

A evapotranspiração média do capim Mombaça, sob sistema de pastejo rotacionado no período de outono/inverno, foi de $3,93 \mathrm{~mm} \mathrm{dia}^{-1}$, com desvio padrão de $1,81 \mathrm{~mm}$.

A determinação da evapotranspiração pelo método do balanço de energia mostrou-se eficiente para o capim Mombaça sob sistema de pastejo rotacionado, porém não foi capaz de determinar a variação da evapotranspiração para cada ciclo de pastejo e sim da área de pasto como um todo.

\section{AGRADECIMENTOS}

Os autores expressam seus agradecimentos ao Conselho Nacional de Desenvolvimento Científico e Tecnológico - CNPq e aos revisores anônimos pelas sugestões.

\section{REFERÊNCIAS BIBLIOGRÁFICAS}

ALENCAR, C. A. B. et al. Irrigação de pastagem: atualidade e recomendações para uso e manejo. Revista Brasileira de Zootecnia, v.38, p.98-108, 2009.
ANDRÉ, R. G. B., MENDONÇA J. C., MARQUES V. S., PINHEIRO F. M.A., MARQUES J. Aspectos energéticos do desenvolvimento da cana-de-açúcar. Parte 1: balanço de radiação e parâmetros derivados. Revista Brasileira Meteorologia, v.25, n.3, p.375-382, 2010.

BIUDES, M. S. et al. Estimativa da evapotranspiração numa pastagem mista, em condições de cerrado, pelos métodos de razão de Bowen e Penman-Monteith. Ciência e Natura, v.30, n.1, 2008.

BOWEN, I. S. The ratio of heat losses by conduction and by evaporation from any water surface. Physical Review, v. 27, n. 6, p. 779-787, 1926.

BROICH, M. et al. Remotely sensed forest cover loss shows high spatial and temporal variation across Sumatera and Kalimantan, Indonesia 2000-2008. Environmental Research Letters, v.6, n.1, p.9, 2011.

CERBU, G. A.; SWALLOW, B. M.; THOMPSON, D. Y. Locating REDD: A global survey and analysis of REDD readiness and demonstration activities. Environmental Science \& Policy, v. 14, n.2, p.168-180, 2011.

DELGADO-ROJAS, J. S. et al. Evapotranspiração máxima do capim "Tanzânia" (Panicum maximum J.) em pastejo rotacionado, baseada na evaporação do tanque "Classe A “ e no índice de área foliar. Engenharia Agrícola, v.4, n.1, p.226-234, 2004.

DIAS-FILHO, M. B.; ANDRADE, C. M. S. Pastagens no ecossistema do trópico úmido. SIMPÓSIO SOBRE PASTAGENS NOS ECOSSISTEMAS BRASILEIROS, v.2, p.95-104, 2005.

GALLARDO, A.; BOND, A. Capturing the implications of land use change in Brazil through environmental assessment: Time for a strategic approach? Environmental Impact Assessment Review, v.31, n.3, p.261-270, 2011.

GUO, Y.; SUN, L. Surface energy fluxes and control of evapotranspiration from a Carex lasiocarpa mire in the Sanjiang Plain, Northeast China. International Journal of Biometeorology, p.1-12, 2011.

HEILMAN, J. L.; BRITTIN, C. L.; NEALE, C. M. U. FETCH REQUIREMENTS FOR BOWEN-RATIO MEASUREMENTS OF LATENT AND SENSIBLE HEAT FLUXES. Agricultural and Forest Meteorology, v.44, n.3-4, p.261-273, 1989.

LAL, M. Implications of climate change in sustained agricultural productivity in South Asia. Regional Environmental Change, v.11, p.S79-S94, 2011.

LIMA, J. R. D. et al. Energy balance and evapotranspiration in cowpea under rainfed conditions. Revista Ciencia Agronômica, v.42, n.1, p.65-74, 2011.

MENDONCA, E. A.; DANTAS, R. T. Estimativa da evapotranspiração de referência no município de Capim, PB. 
Revista Brasileira de Engenharia Agrícola e Ambiental, v.14, n.196-202, 2010.

MENDONÇA, J. C. et al. Determinação do coeficiente cultural (Kc) do feijoeiro (Phaseolus vulgaris L.), em Campos dos Goytacazes, RJ. Revista Brasileira de Engenharia Agrícola e Ambiental, v.11, n.5, p.471-475, 2007.

MENGU, G. P. et al. Impact od climate change on irrigated agriculture. Fresenius Environmental Bulletin, v.20, n.3A, p. 823-830, 2011.

MURRAY, F. W. On the computation of saturation vapor pressure. RAND Corporation. Canta Mônica, CA, p.9. 1967

NUSBAUM, N. J. Dairy Livestock Methane Remediation and Global Warming. Journal of Community Health, v.35, n 5, p.500-502, 2010.

PEDREIRA, M. S. et al. Aspectos relacionados com a emissão de metano de origem ruminal em sistemas de produção de bovinos. Archives of Veterinary Science, v.10, n.3, p.2535, 2005.

RIBEIRO, E. G. et al. Influência da irrigação, nas épocas seca e chuvosa, na produção e composição química dos capins napier e mombaça em sistema de lotação intermitente. Revista Brasileira Zootecnia, v.38, n.8, p.1432-1442, 2009.

RIVERO, S. et al. Pecuária e desmatamento: uma análise das principais causas diretas do desmatamento na Amazônia.

Nova Economia, v.19, n.1, p.41-66, 2009.
SHI, T. T. et al. Comparison of methods for estimating evapotranspiration rate of dry forest canopy: Eddy covariance, Bowen ratio energy balance, and PenmanMonteith equation. Journal of Geophysical ResearchAtmospheres, v.113, n. D19, 2008.

SILVA, L. D. B.; FOLEGATTI, M. V.; VILLA NOVA, N. A. Evapotranspiração do capim Tanzânia obtida pelo método de razão de Bowen e lisímetro de pesagem. Engenharia Agrícola [online], v. 25, n. 3, p. 705-712, 2005. ISSN 0100-6916.

SILVA, T. G. F. et al. Variação do balanço de radiação e de energia da cana-de-açúcar irrigada no semiárido brasileiro. Revista Brasileira de Engenharia Agrícola e Ambiental, v.15, n.2, p.139-147, 2011.

SPAROVEK, G. et al. A revisão do Código Florestal brasileiro. Novos estud. - CEBRAP, n. 89, p.111-135, 2011.

ÜNLÜ, M.; KANBER, R.; KAPUR, B. Comparison of soybean evapotranspirations measured by weighing lysimeter and Bowen ratio-energy balance methods. African Journal of Biotechnology, v.9, n.30, p.4700-4713, 2010.

ZHOU, Y. et al. Impact of climate change on irrigation requirements in terms of groundwater resources. Hydrogeology Journal, v. 18, n. 7, p. 1571-1582, Nov 2010. ISSN 1431-2174. 